\title{
Greffes intrathymiques, ou comment tromper l'adversaire
}

\section{Les nouvelles de ce numéro ont été préparés par Pascale Briand Jean-Claude Dreyfus Jean-Pierre Grünfeld Axel Kahn Claude Matuchansky Marc Peschanski}

1. Posselt AM, Barker CF, Tomaszewski JE, Markmann JF, Choti MA, Naji A. Induction of donor-specific unresponsiveness by intrathymic islet transplantation. Science $1990 ; 249$.
Les services de renseignement connaissent bien la tactique appelée "intoxication ", qui consiste à persuader l'adversaire de la véracité d'une information fausse. C'est un peu ce que viennent de réussir Ali Naji et son équipe de Philadelphie (PA, USA) en déjouant la vigilance des systèmes immunitaires de rejet de greffe par l'implantation du greffon dans le thymus. L'idée sous-jacente à cette expérience est que le thymus est chargé de l'éducation des cellules immunitaires de l'organisme, leur apprenant à distinguer le soi du non-soi. Cela est normalement réalisé par présentation des antigènes du soi à des lymphocytes thymiques en cours de différenciation, de phénotype $\mathrm{CD} 4+, \mathrm{CD} 8+, \mathrm{TcR} / \mathrm{CD} 3+$. Les populations de thymocytes réagissant fortement avec ces antigènes subiront une délétion clonale, comme cela a été discuté en détail maintes fois dans $\mathrm{m} / \mathrm{s}\left(\mathrm{m} / \mathrm{s}\right.$, suppl. au $n^{\circ} 1$, vol. 5, p. 25 ; $n^{\circ} 10$, vol. 5 , p. $788 ; n^{\circ} 2$, vol. 6 , p. 164).

Dans certain cas, les cellules autoréactives seront inactivées sans être détruites (phénomène d'anergie, $\mathrm{m} / \mathrm{s} n^{\circ} 2$, vol. 6, p. 164).

Par conséquent, si on induit une repopulation de l'organisme par des thymocytes fraîchement différenciés chez un animal auquel un tissu étranger a été implanté dans le thymus, on peut supposer que ces nouvelles cellules $\mathrm{T}$ auront été éduquées à ne pas rejeter le greffon. C'est bien ce qu'observent Posselt et al. chez des rats diabétiques subissant une implantation intrathymique d'îlots de Langerhans. Lorsque ce traitement est associé à une injection de sérum antilymphocytaire, qui provoque une déplétion profonde des lymphocytes circulants, les îlots implantés dans le thymus ont, dans 10 expériences sur 13 , une durée de vie dépassant 200 jours et la glycémie est normalisée à long terme. Mieux, même, cette tolérance au greffon intrathymique est associée à la tolérance à un greffon syngénique transplanté ultérieurement, ce qui est attendu si le mécanisme en cause est une éducation de novo des thymocytes au contact des îlots. Ces résultats sont extrêmement importants sur un plan théorique et sur un plan pratique, car ils démontrent que l'éducation intrathymique peut se poursuivre chez l'adulte et ouvrent la perspective à de nouvelles voies thérapeutiques dans le domaine des greffes. On peut, en effet, imaginer qu'une implantation intrathymique d'un fragment d'un organe greffé par ailleurs en position normale (cœur, foie, rein, intestin) puisse extraordinairement améliorer la tolérance immunitaire au greffon. Cette tolérance pourrait-elle aller jusqu'à autoriser des xénogreffes ? Rien ne permet de l'affirmer à cette heure, naturellement. En un temps où l'augmentation régulière des indications de greffe n'est évidemment pas corrélée à l'augmentation de la disponibilité d'organes à greffer, l'utilisation possible d'organes animaux constituerait une immense révolution thérapeutique.

On n'en est pas là, bien sûr, et l'étape suivante sera plus modestement de vérifier s'il est possible d'obtenir de semblables résultats sur de gros mammifères (porc, chien...) avant de passer éventuellement au traitement d'hommes diabétiques. 